\title{
Information's Super-Speed and Adjustable Convey with Matter Wave's Non-Dispersive Propagation
}

\author{
Xinye Wang \\ Retired Scientist \\ Email: wang_xinye@yahoo.com
}

\section{Keywords}

matter wave, wave-particle duality, group velocity, super-speed and adjustable convey

\begin{abstract}
The Wave-Particle Duality is a basic property of microscopic particles. As a basic concept of quantum mechanics, the wave-particle duality theory from elementary particles to big molecules had been verified by lots of experiments. Different from electromagnetic wave, the matter wave's propagation is not only fast but also adjustable. The group velocity with which the overall envelope shape of the wave, namely the related particle's propagation and information convey speed is changeable with its wavelength. When the particle's energy and wavelength, in its non-dispersive propagation, reach to definite values, the group velocity can turn to very high and perhaps is possible to exceed over the light speed in vacuum. Take electron as an example, if the free electron beam gains energy higher than around $4.094 \times 10^{-14} \mathrm{~J}$ and wavelength shorter than around $2.426 \times 10^{-3} \mathrm{~nm}$, the group velocity could surpass the light speed in vacuum. According to the special relativity theory, the light speed in vacuum is the upper limit in the Universe and no matter can exceed over that. As the de Broglie hypothesis and the deduction in this paper do not take relativity theory as premise, the conclusion in this paper should be universality. Wish this paper could provide a different viewpoint for exploration of some scientific problems, such as Faster-Than-Light movement, quantum entanglement mechanism and so on.
\end{abstract}

Wave-Particle Duality is a basic property of microscopic particles. Inspired by Albert Einstein's light wave-particle duality theory, Louis de Broglie in 1924 proposed that all particles exhibit wave-particle duality. This is referred as matter wave and also called as de Broglie wave. (I) This hypothesis was first confirmed by George Paget Thomson's cathode ray diffraction experiment in 1927 and then The Fullerrene molecule in $1999,^{(2)}$ the Phthalocyanine molecule (consist of 58 atoms) in $2012^{(3)}$ and even the big molecule with 25,000amu in $2019^{(4)}$ were discovered in succession with wave-like behaviours. 
According to the wave-particle duality theory, de Broglie combined the Einstein mass- energy equation $\mathrm{E}=\mathrm{mc}^{2}$, one base of the relativity theory related to mass and energy, with the Planck relation $\mathrm{E}=\mathrm{h} v$, one base of quantum mechanics theory related to wave frequency and energy, then equalized both to: $m c^{2}=h v$. However, de Broglie considered that not all matter move with the velocity of light speed in vacuum, so he changed the mass- energy equation from $E=m c^{2}$ to $E=m v^{2}$ by replacing $c$ with the actual velocity $v$. and equal both as $m v^{2}=h v$.

This important change means that the de Broglie hypothec did not take the relativity theory as its theoretical base and therefore the particle's moving velocity is not restricted. This newly de Broglie hypothec was formulated as below:

$\mathrm{mv} \mathrm{v}^{2}=\mathrm{h} v$

As $v=v / \lambda, p=m v$, thus

$\mathrm{p}=\mathrm{h} / \lambda$

Formula (1), (2) also can be written as

$p=\hbar k$

$E=\hbar \omega$

Among formula (1),(2),(3)and(4), $v$ is particle velocity, $v$ is wave frequency, $p$ is momentum, $E$ is energy, $\lambda$ is wavelength, $k$ is angular wavenumber, $\omega$ is angular frequency, $h$ is Planck constant and $\hbar$ is reduced Planck constant.

The group velocity of a wave is the velocity with which the overall envelope shape of the wave's amplitudes -known as the modulation or envelope of the wave propagates through space and is often thought of as the velocity at which energy or information is conveyed along a wave. The group velocity, $v g$ is defined by equation (5)

$\operatorname{vg}=\frac{\partial \omega}{\partial \kappa}=\frac{\partial \mathrm{E}}{\partial \mathrm{p}}$

Matter wave's non-dispersive propagation means its propagation in space without effects either by interaction with transmitting medium or by geometric boundary condition.

The matter wave's propagation can be classified into two types: relativistic propagation and de Broglie's propagation-Free Particle's Non-Relativistic Propagation:

\section{0) Relativistic Propagation:}

Particle's total energy E, rest(intrinsic) mass $m$, momentum $p$, light speed in vacuum $c$ related as:

$E^{2}=\left(m c^{2}\right)^{2}+(p c)^{2(5)}$ 


\section{1) rest mass $m=0, E=p c$ thus}

$\mathbf{v g}=\frac{\partial E}{\partial \mathbf{p}}=\mathbf{c}$

This is the group velocity of light, namely the photon's speed in vacuum.

1.2) rest mass $m \neq 0$, particle's speed approaches to light speed in vacuum-ultrarelativistic limit, as pc $» \mathrm{mc}^{2}$, so $\mathrm{E}=\mathrm{pc}$. thus

$\mathrm{vg}=\frac{\partial \mathrm{E}}{\partial \mathbf{p}}=\mathrm{c}$

This is the group velocity of related particle, which moves with the light speed in vacuum.

1-3) rest mass $m \neq 0$ and particle's speed less than $c$ in the nonrelativistic limit,

$E=m c^{2}+\frac{p^{2}}{2 m}$

In formula (8), $p=m v$. According to author's estimate, item $\mathrm{mc}^{2}$ is less than item $\frac{\mathrm{p}^{2}}{2 \mathrm{~m}}$ by around two orders of magnitude. Whether or not to make approximate assumption to formula (8), as value of $\mathrm{mc}^{2}$ is invariant, the result is the same as:

$v g=\frac{\partial E}{\partial p}=\frac{p}{m}=\frac{\hbar k}{m}$

\section{0) De Broglie's Propagation-Free Particle's Non Relativistic Propagation}

De Broglie concluded that the velocity of a particle should always be equal to the group velocity of the corresponding wave. This conclusion can be proved as below:

As the total energy for a free particle in non-relativistic propagation is

$E=\frac{p^{2}}{2 m}$

So

$v g=\frac{\partial E}{\partial p}=\frac{p}{m}$

In formula (10) $\mathrm{p}=\mathrm{mv}$, thus

$\operatorname{vg}=\frac{\partial E}{\partial p}=\frac{p}{m}=V$

From (11), the de Broglie's conclusion proved, then from formula (10)

$\operatorname{vg}=\frac{\partial E}{\partial p}=\frac{p}{m}=\frac{\hbar k}{m}=\frac{h}{m \lambda}$ 
The formula (12) indicates that the group velocity vg is changeable with wavelength $\lambda$, and the value of vg is unlimited. When the wavelength $\lambda$ reduces to certain extent, the group velocity, vg can increase much and even exceed over the light speed in vacuum.

Suppose

$\lambda \leq \frac{h}{\mathrm{mc}}$

$\mathbf{E}=\frac{\mathbf{p}^{2}}{2 \mathbf{m}} \geq \frac{\mathbf{h}^{2}}{2 \mathbf{m} \lambda^{2}}$

Thus

$\mathbf{v g}=\frac{\hbar \kappa ́}{\mathbf{m}} \geq \frac{h}{\mathbf{m} \boldsymbol{\lambda}}$

If the matter wave's particle is electron, $m_{e}, \lambda_{e}, k_{e}, v_{e}$ represent its mass, wavelength, angular wavenumber and group velocity. As $m_{e}=9.109 \times 10^{-31} \mathrm{~kg}, \mathrm{~h}=6.62607015 \times 10^{-34} \mathrm{~J} . \mathrm{s}$, $\mathrm{c}=299,792,458 \mathrm{~m} \cdot \mathrm{s}^{-1}$, so

$\lambda_{\mathrm{e}} \leq \frac{\mathrm{h}}{\mathrm{m}_{\mathrm{e}} \mathrm{c}}=2.426 \times 10^{-12} \mathrm{~m}=2.426 \times 10^{-3} \mathrm{~nm}$

$E_{e}=\frac{p_{e}^{2}}{2 m} \geq \frac{h^{2}}{2 m_{e} \lambda_{e}^{2}}=4.094 \times 10^{-14} \mathrm{~J}$

And

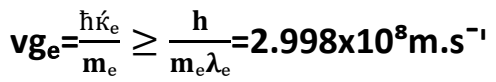

Formula (16), (17) indicate that when electron's wavelength is shorter than $2.426 \times 10^{-3} \mathrm{~nm}$ and electron's energy is higher than $4.094 \times 10^{-14} \mathrm{~J}$, the electron's propagation speed (18) is exceed over the light speed in vacuum.

This result means that the matter wave is quite different from the electromagnetic wave. Although both propagate information, the convey velocity for matter wave is adjustable and even able to faster than that of light in vacuum, while the convey velocity of electromagnetic wave is generally equal to the light speed and almost unchangeable.

\section{Facts and Data}

The electron microscope uses a beam of accelerated electrons under a very high voltage of several $10^{3}$ to $3 \times 10^{6}$ voltages (the HVEM can be applied to 200 to $1000 \mathrm{kV}$ ) between a cathode, as an electron source and an anode, as an acceleration part, going through a vacuum area to illuminate the specimen and create an image. As the wavelength of electron can be up to 100,000 times shorter than that of visible light photons, electron microscopes have higher resolving power than light microscopes and can reveal the ultrastructure of smaller objects. 
If the electron beam is accelerated by anode with voltage of $3 \times 10^{2} \mathrm{kV}$, i.e. $\mathrm{V}=3 \times 10^{5} \mathrm{~V}$, as electron's charge, $e=1.602 \times 10^{-19} \mathrm{C}$, electron's mass, $m_{e}=9.109 \times 10^{-31} \mathrm{~kg}$, Planck constant, $\mathrm{h}=6.626 \times 10^{-34} \mathrm{~J} . \mathrm{s}$, the electron's energy accelerated , $E_{e}$, wavelength, $\lambda_{e}$ and group velocity, $v_{e}$ should equal to:

$E_{e}=e V=4.806 \times 10^{-14} \mathrm{~J}$

For free particle,

$E_{e}=\frac{p_{e}^{2}}{2 m}=\frac{h^{2}}{2 m_{e} \lambda_{e}^{2}}=4.806 \times 10^{-14} \mathrm{~J}$

Thus

$\lambda_{\mathrm{e}}=2.239 \times 10^{-12} \mathrm{~m}=2.239 \times 10^{-3} \mathrm{~nm}$

$v_{\mathrm{e}}=\frac{\mathrm{h}}{\mathrm{m}_{\mathrm{e}} \lambda_{\mathrm{e}}}=3.248 \times 10^{8} \mathrm{~m} \cdot \mathrm{s}^{-1}$

The results of (19), (20) and (21) is consistent with the formula (17), (16) and (18).

\section{Conclusion}

Different from electromagnetic wave, the matter wave's propagation, that is, the related particle's movement and information's convey is not only fast but also adjustable. The velocity can be changed with its wavelength and In the non- dispersive propagation, it can reach to very high speed and is able to exceed over the light speed in vacuum. Take electron as an example, the electron's propagation and related information's convey speed could surpass the light speed in vacuum, if its wavelength is shorter than around $2.426 \times 10^{-3} \mathrm{~nm}$ and its energy is higher than around $4.094 \times 10^{-14} \mathrm{~J}$ in the non- dispersive propagation. As the de Broglie hypothesis did not take relativity theory as premise and the deduction in this paper also proceeds under non-relativity condition, the conclusion in this paper should be universality. According to Special Relativity Theory, the light speed in vacuum is the upper limit in the Universe and no matter can exceed over that. Wish this paper could provide a different viewpoint to understand some scientific problems such as Faster-Than-Light movement, quantum entanglement mechanism and so on.

The author: Wang Xinye graduated from Tongji University, Shanghai, China in 1964 and now is a retired senior engineer (in China) and a retired scientist (in Singapore)

\section{References}

(1) de Broglie,L Nobel Lecture:The Wave Nature of the Electron. The Nobel Foundation.1929 (2008-

08-30)

(2) L'Anmunziata,Michael,Radiactivity:introduction and History,From the Quantum to Quarks 2nd,Elsevier Science,2016

(3) Juffmann,Thomas, Real-time single-molecule imaging of quantum interence. Nature

(4) phys org. Retrieved 25 September 2019. Nanotechenology 25 March2012

(5) Taylor:Classical Mechanics.University Science Books:652.ISBN 1-891389-22-X 

